\title{
Highlights from the San Antonio Breast Cancer Symposium (SABCS) 2019
}

\author{
Isabell Witzel $^{\mathrm{a}}$ Matteo Lambertini ${ }^{\mathrm{b}, \mathrm{c}}$ Marija Balic $^{\mathrm{d}}$ Rachel Wuerstlein $^{\mathrm{e}}$ \\ Volkmar Müllera

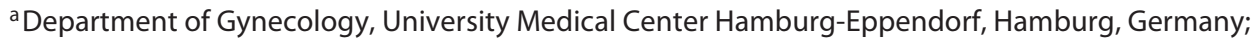 \\ ${ }^{b}$ Department of Medical Oncology, U.O.C. Clinica di Oncologia Medica, IRCCS Ospedale Policlinico San Martino, \\ Genova, Italy; ${ }^{C}$ Department of Internal Medicine and Medical Specialties (DiMI), School of Medicine, University \\ of Genova, Genova, Italy; ${ }^{d}$ Klinische Abteilung für Onkologie, Universitätsklinik Graz, Graz, Austria; ${ }^{\mathrm{e}}$ Department \\ of Obstetrics and Gynecology, Breast Center and Comprehensive Cancer Center (CCC Munich LMU), University \\ Hospital, LMU Munich, Munich, Germany
}

\section{Question 1: From Your Point of View, Which Data Presented at This Year's San Antonio Breast Cancer Symposium 2019 Would You Consider as the Most Practice-Changing Results?}

Lambertini: The phase III data of the HER2CLIMB trial simultaneously published in the New England Journal of Medicine are definitively the most practice-changing results [1]. This study has clearly demonstrated that the addition of tucatinib (which is a highly selective inhibitor of the HER2 tyrosine kinase) to trastuzumab and capecitabine represents an effective treatment option for heavily pretreated patients with HER2-positive advanced breast cancer (one-third reduction in the risk of death, halved risk of progression or death, and doubled objective response rate). Importantly, almost half of the patient population included in the trial had presence/history of brain metastases, which represent an important unmet medical need for which limited effective treatment options are available. Key safety adverse events were diarrhea, liver transaminase elevations, and palmar-plantar erythrodysesthesia, being of low grade in the majority of the cases and leading to discontinuation of the experimental drug in only $6 \%$ of the patients.
Müller: HER2CLIMB was also for me the most practice-changing study. So far, no study has evaluated the treatment standard after first-line treatment with trastuzumab/pertuzumab and further-line treatment with T-DM1. One additional aspect of high clinical relevance is the fact that almost half of the patients had brain metastases, a situation with high unmet clinical need. In San Antonio and in the simultaneous full publication, there was a similar benefit for the progression-free survival of these patients. I am anxiously waiting for more detailed upcoming analyses in this cohort concerning brain metastases response and clinical outcome of these patients.

Balic: Also in my view, in the first general session of the San Antonio Breast Cancer Symposium (SABCS), a selection of very important studies was presented.

Besides the HER2CLIMB trial, the trial DESTINYBreast01 provided very important supporting evidence for trastuzumab-deruxtecan in heavily pretreated patients with HER2-positive metastatic breast cancer with a median number of 6 treatment lines [2]. The efficacy of the treatment was great, and in a great majority of patients, tumor shrinkage could be induced. The FDA has already granted an accelerated approval, and the hope is 
that this drug will soon be available also in Europe for patients despite the necessary caution regarding the lung interstitial disease occurring in more than $10 \%$ of treated patients.

In the presentation of the plasmaMATCH trial, interesting results were shown, demonstrating a relevant clinical application for cfDNA-based mutation analysis for the selection of the treatment approach. For 2 selected populations and drugs, positive results have been reported, as well as HER mutations and treatment with neratinib and AKT mutations and treatment with capivasertib.

Wuerstlein: I also think that the results of 3 new agents for metastatic HER2-positive breast cancer that were presented at SABCS - and 2 of them simultaneously published - will change our practice: tucatinib, margetuximab, and trastuzumab-deruxtecan.

Data from HER2CLIMB not only gives significant results on overall survival with the combination of tucatinib, capecitabine, and trastuzumab, but also fits our standard of care concerning therapy sequencing after the use of T-DM1 and showed great results for brain metastases, even active brain metastases (1-year progressionfree survival, 25 vs. $0 \%$ in the control arm), and good data for safety management.

Trastuzumab-deruxtecan, as shown in the DESTINYBreast0 1 trial, is a new antibody drug conjugate and again active after progression with T-DM1 and even in heavily pretreated patients. This phase II trial shows good data with an overall response rate of $60 \%$. DESTINY trials are now open and a great option for our patients.

\section{Question 2: Do You Think that This Data Should Be Transferred into Clinical Routine Immediately? And if You Think so: How Should This Be Done?}

Wuerstlein: Several trials are now open in Europe that will be an option for our patients to get early access to these new drugs. In the meantime, regulatory standards will be followed. Options in centers offering precision medicine or molecular tumor boards may be another way to early access, as shown for margetuximab and CD16 phenotype.

Müller: The availability of tucatinib in clinical routine would allow the transfer into routine use.

Lambertini: The results of this trial should be transferred into clinical routine as soon as the drug tucatinib is approved. The combination of tucatinib plus trastuzumab plus capecitabine should then become a standard treatment option in patients with HER2-positive advanced breast cancer previously treated with trastuzu- mab, pertuzumab, and T-DM1. Importantly, this therapy will also become an important treatment option for patients with brain metastases. Based on the solid data presented, highly applicable to the current population of patients treated nowadays for HER2-positive advanced breast cancer, this could become the preferred third-line option, following pertuzumab-based first-line therapy and second-line T-DM1. Notably, the evidence on the activity and efficacy of the other available treatment options in this setting has been obtained before the introduction of pertuzumab and T-DM1.

Balic: Tucatinib is expected to enrich the portfolio of possibilities for the treatment of HER2-positive disease soon. Based on the data of DESTINY-Breast01, it should lead to a rather quick implementation of trastuzumabderuxtecan in pretreated patients, and we also hope that studies will prove its usefulness in the earlier setting. For both of these studies, regulatory approval will be followed by regular clinical implementation. Before that, in selected centers, early access programs will hopefully be implemented. The plasmaMATCH trial will provide a possibility for selected sites where testing is available, as well as for selected patients. This approach will most probably only be available in larger centers.

\section{Question 3: What Is Your Personal Opinion Regarding the Data Presented with Regard to Immunotherapy in Triple-Negative Breast Cancer - and Do You See a Role for Immunotherapy also in Other Subtypes?}

Lambertini: Conflicting results have been reported with the use of immunotherapy in triple-negative breast cancer. At the present time, immunotherapy (atezolizumab) in combination with chemotherapy (nab-paclitaxel) is standard of care only as first-line therapy in patients with triple-negative PD-L1-positive metastatic breast cancer.

In the neoadjuvant setting, updated results from the KEYNOTE-522 trial have shown that the addition of pembrolizumab to neoadjuvant chemotherapy (consisting of carboplatin plus paclitaxel followed by doxorubicin/epirubicin plus cyclophosphamide) was associated with a significant improvement in the rates of pathologic complete response (pCR) in patients with triple-negative early breast cancer. The benefit appeared larger in patients with high disease burden (stage III and/or node positive). However, in a similar population of patients with triple-negative early breast cancer, the NeoTRIPaPDL1 trial did not show any improvement in the $\mathrm{pCR}$ rate with the addition of atezolizumab to neoadjuvant chemotherapy (consisting of carboplatin plus nab-paclitaxel). In 
the SAFIR02 trial, maintenance single-agent immunotherapy with durvalumab was not superior to chemotherapy in patients with HER2-negative metastatic breast cancer without targetable molecular alterations and not progressing after 6-8 cycles of chemotherapy. However, exploratory analyses in the triple-negative and PD-L1positive populations suggested improved overall survival for patients exposed to durvalumab. Overall, these data suggest that more biomarker-driven research efforts are needed in the breast cancer field to optimize the use of immunotherapy (i.e., patient selection).

Müller: I consider the SAFIR02 trial as not conclusive as I do think the study concept does not allow any conclusions for the clinical routine. Despite of the negative outcome of the NeoTRIPaPDL1 trial with a non-anthracycline neoadjuvant chemo backbone, I am optimistic that the increase of pCR rate seen in the KEYNOTE-522 trial with the addition of pembrolizumab to neoadjuvant chemotherapy will also transfer to an improvement in outcome. Considering the side effects of immunotherapy, I would wait for a more definite analysis of disease-free survival as the results presented were in my view preliminary.

Balic: There is still much to be understood, even in the potentially most immunogenic subtype of breast cancer. However, it seems that, in the setting of high-risk triplenegative breast cancer with larger tumors and lymph node positivity, the integration of immune checkpoint inhibitors in the early-stage treatment may be faster. Before these compounds are widely implemented, though, more positive studies and confirmatory data as well as longer follow-up will be needed. In other subtypes, particularly in HER2-positive breast cancer, there is a potential, but still a long way to go. In ER-positive breast cancer, a better understanding and more effective combinations with immunomodifying agents are needed as well, and studies addressing this question are being designed and initiated. The most effective immunostimulators are to be defined and confirmed in larger trials, including chemotherapy, radiotherapy, diverse targeted therapies, and other interesting combinations, including other immune checkpoint inhibitors.

Wuerstlein: We will learn about the use of immunotherapy in high-risk luminal breast cancer and HER2positive breast cancer with the data from ongoing trials.

At SABCS, data from 2 agents in triple-negative breast cancer (pertuzumab and atezolizumab) were presented with new subgroup data in KEYNOTE-522 and first data for atezolizumab in the neoadjuvant setting.

For various reasons, these trials cannot be compared. They had different trial designs and chemotherapy back- bone, and one agent was a PD-1 inhibitor, while the other was a PD-L1 inhibitor.

Whether checkpoint inhibitors will be a new standard of care in the neoadjuvant setting of breast cancer is a question to answer over the next months. Right now, checkpoint inhibitors show convincing data in clinical high-risk patients, justifying also the short- and longterm toxicities of these drugs.

\section{Question 4: Which Result Do You Consider Surprising (either in a Positive or a Negative Way)?}

Lambertini: The most surprising results, but eagerly awaited after the promising phase I data, were those of the DESTINY-Breast01 study simultaneously published in the New England Journal of Medicine. This is a phase II trial that investigated the activity of DS-8201 (trastuzumab-deruxtecan), a new potent antibody-drug conjugate composed of an anti-HER2 antibody linked to a cytotoxic topoisomerase I inhibitor, in pretreated patients (including all patients previously exposed to T-DM1) with advanced HER2-positive breast cancer. In a heavily pretreated patient population (median number of prior lines equal to 6, ranging from 2 to 27), the study has shown an impressive objective response rate of $60.9 \%$ (95\% CI $53.4-68.0)$, clinical benefit rate at 6 months of $76.1 \%$ (95\% CI 69.3-82.1), and progression-free survival of 14.8 months (95\% CI 13.8-16.9). The most common side effect was nausea, of grade 1-2 in the majority of the cases. Notably, $13.6 \%$ of the patients developed interstitial lung disease being of grade 5 in $4(2.2 \%)$ of the patients. Phase III trials with the use of this agent are already ongoing and may radically change the treatment algorithm for HER2positive advanced breast cancer.

Müller: My positive surprise were also the DESTINY results which (if confirmed in the ongoing phase III studies) would be another very potent drug for HER2-postive breast cancer. I am a little disappointed by the APHINITY results showing a moderate benefit in many subgroups with a good outcome in the overall cohort. In my opinion, these results support the concept of neoadjuvant treatment with treatment escalation in the nonresponding patients and (hopefully) also approaches for de-escalation in responding patients.

Balic: For me, the updated results of the adjuvant APHINITY trial were rather positively surprising, particularly in the group of ER-positive HER2-positive patients receiving pertuzumab in addition to trastuzumab for 1 year. After 6 years of follow-up, the outcome became positive in this subgroup of patients as well, with a $3 \%$ absolute difference in invasive disease-free survival and a 
hazard ratio of 0.73 . Somewhat negatively surprising were the results of the NeoTRIP trial (neoadjuvant trial adding atezolizumab to carboplatin and nab-paclitaxel in triplenegative breast cancer). However, the role of immunotherapy is still not well understood, and results still do not allow for any definitive conclusions.

Wuerstlein: I was positively surprised that a lot of important data for metastatic breast cancer was presented this year in contrast to previous years. However, the pCR rate in the NeoTRIP trial was rather disappointing (43.5 vs. $40.8 \%)$.

\section{Question 5: Were There Any Interesting Data Concerning Oral Therapies in the Adjuvant Setting (e.g., Endocrine Treatment)?}

Balic: Interesting novel data regarding oral adjuvant therapies are expected, and at the moment, there are no data impacting the clinical routine. The data on adjuvant CDK4/6 inhibitors or PARP inhibitors have not yet been reported and are eagerly awaited.

Müller: Results from the EBCTCG overview group have examined the changes in long-term outcome of hormone receptor-positive breast cancer patients. Based on the results presented, it can be expected that the longterm outcome was improved by approximately $25 \%$ with an overall recurrence rate of $10 \%$ in the years 5-20 after diagnosis.

Wuerstlein: In addition to the EBCTCG meta-analysis, NSABP-42 data proved the extended use of endocrine treatment over 10 years in selected patients with luminal subtype. This confirms our recommendations in the German AGO guidelines (www.ago-online.de).

First data for use of CDK4/6 inhibitors (for 6 months) in the neoadjuvant setting of luminal B type early breast cancer showed promising results and a better safety profile in comparison to chemotherapy (CORRALLEE trial).

Lambertini: More data have become available on the role of capecitabine as adjuvant treatment in patients with triple-negative breast cancer. An individual patient data meta-analysis and a new phase III trial (the CBCSG-10 study, despite a not-optimal backbone chemotherapy approach) have provided additional evidence on the benefit associated with the use of capecitabine in this setting. Addition of capecitabine significantly improved disease-free survival in both studies and overall survival only in the individual patient data meta-analysis with a trend in the CBCSG-10 study. Taken together, these data support the use of capecitabine as oral therapy in high-risk triple-neg- ative breast cancer, such as those not achieving a pCR after standard neoadjuvant chemotherapy. Several trials are ongoing to explore the benefit of other treatment options (including single-agent platinum-based chemotherapy and immunotherapy) in this setting.

\section{Question 6: Which Basic Science Data Would You Consider as Being Meaningful for Clinical Practice - either Now or in the Future?}

Balic: With novel technologies and an increasing number of efforts, the biology of breast cancer and the mechanisms of carcinogenesis, invasion, immune escape, metastasis, drug resistance, and other components of disease progression and clinical challenges are being decoded. Presently, first insights have found their way into clinics, and one example is the plasmaMATCH trial. Although cell-free DNA and liquid biopsy are not a part of clinical routine, first clinical data demonstrating benefit for patients with specific mutations detected by targeted sequencing and drug selection based on these assays are promising. In triple-negative breast cancer, it has been shown in a study that cell-free DNA-based assays can distinguish patients at risk for disease relapse. The AURORA trials, such as data presented in San Antonio from the AURORA-US, allow for a very detailed analysis of disease clonality in the primary tumor and various metastatic sites. In the future, these analyses will help define novel therapeutic approaches. Furthermore, novel profiling technologies, as shown in the meeting, may allow for a selection of patients who are the best candidates for selected treatments, including immunotherapy.

Lambertini: Important evidence with potential future clinical implications has been provided about possible mechanisms of intrinsic and acquired resistance to CDK4/6 inhibitors. By analyzing multiple endocrinesensitive and endocrine-resistant breast cancer cell lines, De Angelis et al. [3] have found that high levels of interferon (IFN) signaling were associated with increased resistance to either genetic (by shRNA CDK4 knockdown) or pharmacological(by palbociclib) inhibition of CDK4/6. By evaluating the correlation between the expression of the IFN response genes and cell growth inhibition upon palbociclib treatment in vitro, the authors derived a 35gene signature associated with reduced response to palbociclib (IFN-related palbociclib-resistance signature [IRPS]). Notably, IRPS and other IFN-related signatures were highly enriched in patients from the NeoPalAna and the neoMONARCH neoadjuvant trials with tumors exhibiting intrinsic resistance to CDK4/6 inhibitors. Looking into the gene expression profiles of primary estrogen receptor-positive/HER2-negative breast tumors from the 
TCGA and METABRIC datasets, the IRPS scores were found to be higher in luminal B than luminal A subtype, and patients with high-IRPS tumors had a significantly shorter breast cancer-specific survival compared to patients with low-IRPS tumors, independently from the luminal subtype. Interestingly, in estrogen receptor-positive/HER2-negative tumors, high IRPS scores were highly correlated with the expression of immune checkpoints (PD-L1, PD-1, and CTLA4) and with immune-suppressive regulatory T-cells infiltration. Finally, in breast cancer cell line derivatives with acquired resistance to palbociclib, the authors detected a dramatic activation of the IFN/STAT1 signaling compared to short-term palbociclib-treated or untreated parental cells. Taken together, these data suggest that IFN signaling is associated with intrinsic and acquired resistance to CDK4/6 inhibitors. Therefore, the IFN pathway may serve in the future as a predictive biomarker for response/resistance to CDK4/6 inhibitors and may provide potential novel therapeutic targets for improving the efficacy of CDK4/6 inhibitors and patients' outcome.

Müller: The plasmaMATCH trial examined the use of mutations detected in cfDNA to guide therapy decisions. For me, the overall response rates were not really convincing and I wonder if a control group without detected mutations would not be important. Therefore, this approach might be of relevance in the future but surely has no clinical application outside of trials.

Wuerstlein: Data from the SAFIR02 Breast trial and the plasmaMATCH trial confirm our way to individualize more and more our treatment strategies in metastatic and also early breast cancer.

PD Dr. med. Rachel Wuerstlein

Breast Center, Department of Gynecology and Obstetrics

and CCC Munich, University of Munich (LMU)

Marchioninistr. 15

81377 Munich, Germany

Rachel.Wuerstlein@med.uni-muenchen.de

Prof. Dr. med. Volkmar Müller

Konservative gynäkologische Onkologie

Klinik und Poliklinik für Gynäkologie

Universitätsklinikum Hamburg-Eppendorf

Martinistrasse 52

20246 Hamburg, Germany

vmueller@uke.uni-hamburg.de

\section{Disclosure Statement}

Matteo Lambertini served as a consultant for Roche and received speaker's honoraria from Theramex, Roche, and Takeda outside the submitted work.

Volkmar Müller received speaker honoraria from Amgen, Astra Zeneca, Celgene, Daiichi-Sankyo, Eisai, Pfizer, MSD, Novartis, Roche, and Teva and consultancy honoraria from Genomic Health, Hexal, Roche, Pierre Fabre, Amgen, ClinSol, Novartis, MSD, Daiichi-Sankyo, Eisai, Lilly, Tesaro, and Nektar as well as institutional research support from Novartis, Roche, Seattle Genetics, and Genentech.

Rachel Wuerstlein received speaker honoraria from Agendia, Amgen, Aristo, Astra Zeneca, Boeringer Ingelheim, Carl Zeiss, Celgene, Clinsol, Daiichi-Sankyo, Esai, Genomic Health, Glaxo Smith Kline, Hexal, Lilly, Medstrom Medical, MSD, Mundipharma, Nanostring, Novartis, Odonate, Paxman, Palleos, Pfizer, Pierre Fabre, PumaBiotechnolgogy, Riemser, Roche, Sandoz/ Hexal, Seattle Genetics, Tesaro Bio, Teva.

Marija Balic received consulting fees, lecture honoraria, advisory board memberships, and travel grants from Amgen, AstraZeneca, Bayer, Boehringer Ingelheim, Celgene, Lilly, MSD, Novartis, Pfizer, and Roche, as well as research funding from Eli Lilly and Pfizer.

References

Prof. Matteo Lambertini

Department of Medical Oncology

U.O.C. Clinica di Oncologia Medica

IRCCS Ospedale Policlinico San Martino and

University of Genova

Largo Rosanna Benzi 10

16132 Genova, Italy

matteo.lambertini@unige.it

Prof. Dr. Marija Balic

Division of Oncology, Department of Internal Medicine

Medical University Graz

Auenbruggerplatz 15

8036 Graz, Austria

marija.balic@medunigraz.at

\footnotetext{
1 Murthy RK, Loi S, Okines A, Paplomata E, Hamilton E, Hurvitz SA, et al. Tucatinib, Trastuzumab, and Capecitabine for HER2Positive Metastatic Breast Cancer. N Engl J Med. 2019 Dec, Epub ahead of print.

2 Modi S, Saura C, Yamashita T, Park YH, Kim SB, Tamura K, et al.; DESTINY-Breast01 Investigators. Trastuzumab Deruxtecan in Previously Treated HER2-Positive Breast Cancer. N Engl J Med. 2019 Dec, Epub ahead of print.

3 De Angelis C, Fu X, Cataldo ML, Nardone A, Jansen VM, Veeraraghavan J, et al. High levels of interferon-response gene signatures are associated with de novo and acquired resistance to $\mathrm{CDK} 4 / 6$ inhibitors in ER+ breast cancer. SABCS. 2019;GS 2-01.
} 